\title{
New Aspects of the Non Commutative Space-Time Cosmology
}

\author{
N.Mebarki ${ }^{1}$ \\ Laboratoire de Physique Mathematique et Subatomique, Mentouri University \\ Route Ain El Bey, Constantine 25000, Algeria \\ E-mail: nnmebarki@yahoo.fr
}

New cosmological aspects of the Seiberg-Witten non commutative geometry (NCG) are discussed. Using a Kodama like observer via the tunnelling effect, the NCG black hole Hawking temperature of the apparent horizon in an FRW like model is obtained. Moreover, it is shown that because of the anisotropy generated by NCG, a new mechanism explaining leptogenesis is proposed and the related leptonic asymmetry is calculated.

The 2011 Europhysics Conference on High Energy Physics-HEP2011

Grenoble, Rhône-Alpes France

July 21-27 2011

${ }^{1}$ Speaker 


\section{Introduction}

Recently, the noncommutative nature of the space-time, has been a subject of a very active research[1]-[3]. The motivation lies in the fact that the space-time non commutativity (NCG) could be significant at the Planck scale and very sensitive to a search for signatures in the cosmological observations such as the observed anisotropies of the cosmic microwave background (CMB)[4]-[7]. The goal of this paper is to show the new cosmological aspects of NCG. In section2, we calculate the NCG dynamical black hole Hawking temperature. More important, we propose a new mechanism for leptogenesis. In fact, we prove that despite the initial spherical symmetry that one has in the FRW universe, a pure NCG lepton number asymmetry can be generated. In section3, we draw our conclusions.

\section{Formalism}

Using the NCG FRW like metric of ref.(5) (see eqs.(14)) in spherical coordinates $(r, \theta, \varphi)$ and choice of a power law formula $a=\hat{t}^{\beta}$ for the commutative scale factor and non vanishing noncommutativity parameter $\theta^{12}=\eta$, one can show that the energy of the particles is defined using a Kodama observer characterized by a vector field $K^{\mu}$ having the following components:

$$
K^{\mu}=\left(\left(\Sigma_{3}^{2}-\Sigma_{1} \Sigma_{2}\right)^{-1 / 2}, 0,0,0\right)
$$

where $\hat{t}=t / t_{0}$ is a dimensionless time ( $t_{0}$ is the observed time) and:

$$
\begin{aligned}
& \Sigma_{1}=-\left(1-H_{\hat{t}}^{2} \tilde{r}^{2}\right)+\eta^{2}\left(\Lambda_{1}+\Lambda_{2} H_{\hat{t}}^{2} \tilde{r}^{2}-\Lambda_{3} H_{\hat{t}} \tilde{r} / a_{N C G}\right) \\
& \Sigma_{2}=\left(1-H_{\tilde{r}} \tilde{r}\right)^{2}\left(1+\eta^{2} \Lambda_{2}\right) \\
& \Sigma_{3}=\left(1-H_{\tilde{r}} \tilde{r}\right)\left[+2 \tilde{r} H_{\hat{t}}+\eta^{2}\left(2 \tilde{r} H_{\hat{t}} \Lambda_{2}-\Lambda_{3} / a_{N C G}\right]\right.
\end{aligned}
$$

with

$$
\begin{aligned}
& \Lambda_{1}=-\beta(\beta-1)\left(\hat{t}^{(\beta+1)}+18 \hat{r}^{2} \beta^{3} \hat{t}^{4(\beta-1)}\right) / 32 \\
& \Lambda_{2}=\beta^{2}\left(-18 \hat{t}^{-2}+21 \hat{r}^{2} \beta^{2} \hat{t}^{4(\beta-1)}\right) / 64 \\
& \Lambda_{3}=\beta^{2} \hat{r} \hat{t}^{4 \beta-1}(28-25 \beta) \\
& a_{N C G}=\hat{t}^{\beta}\left[1+\eta^{2} \beta^{2} \hat{t}^{2(\beta-1)}\left(21 \beta^{2} \hat{t}^{2(\beta-1)}-45\right]\right.
\end{aligned}
$$

Here $a_{N C G}, H_{\hat{t}}=\partial a_{N C G} / \partial \hat{t}, H_{\tilde{r}}=\partial a_{N C G} / \partial \tilde{r}, \hat{r}=r / r_{0}$ and $\tilde{r}=a_{N C G} \hat{r}$ are the NCG scale factor, time and space evolution Hubble parameters, dimensionless and reduced radii respectively. Now, within the fermionic tunneling approach and using the Hamilton-Jacobi method together with the WKB approximation leads to the following expression of the noncommutative black hole (NCGBH) Hawking temperature $T_{H}$ :

$$
T_{H}=2 \pi \Sigma_{2}^{1 / 2} /\left(K^{0} \Sigma_{3}\right)
$$

Moreover, taking as a non vanishing component of the noncommutativity parameter $\theta^{23}=\eta$, we can show easily that left and right handed particles propagating in the presence of a non 
commutative curved space-time (initially having a spherical symmetry) have different energies. After straightforward calculations, we deduce the following expression of the dynamical anisotropic leptonic asymmetry $\Delta n_{N C G}$ for the ultrahigh energy particles (neutrinos):

$$
\Delta n_{N C G} \approx \frac{8 g}{(2 \pi)^{2}} T^{3} \sum_{k=1}^{\infty} \frac{(-1)^{k}}{k^{3}} \sinh \left(\frac{k \hat{B}_{0}}{T}\right)
$$

where $g$ (resp. $T$ ) is the particle number of degrees of freedom (resp. the temperature) and:

$$
\hat{B}_{0}=\eta\left[\frac{1}{4} \hat{t}^{\beta-2} \beta^{2} \hat{r}-2 \beta^{2} \hat{t}^{3 \beta-6}+\frac{1}{4} \beta^{2} \hat{r}^{2} \hat{t}^{3 \beta} \sin \theta-\frac{\hat{t}^{-\beta}-2(1+\hat{r}) \cos ^{2} \theta}{2 \hat{r} \sin \theta}+\frac{\hat{t}^{-\beta}}{4 \hat{r}} \cot g^{2} \theta\right]
$$

This is a pure NCG result. As it was pointed out in ref.[8], in order to have this leptonic asymmetry and an energy gap between the particle and antiparticle at the thermodynamic equilibrium, the space-time metric has to have non zero off diagonal spatial components and CPT violating terms in the Lagrangian. In our case, these two conditions are fulfilled (see ref.5 for the NCG metric ) where the NCG not only violates Lorentz invariance( thus, CPT symmetry ) but generates an inhomogeneity and breaks the spherical symmetry as well.

\section{Conclusion}

We have obtained the time and space evolution of the NCG Hawking temperature $T_{H}$ related to the NCGBH apparent dynamical event horizon. The qualitative results show that $T_{H}$ is a decreasing but strongly dependent function of the NCG parameter $\eta$. Moreover, and contrary to the commutative FRW universe with a spherical symmetry, a new mechanism for leptogenesis is proposed and a pure anisotropic NCG dynamical leptonic asymmetry has been obtained in the decoupling temperature limit.

\section{References}

[1]J. P. Kneller, R. J. Scherrer, G. Steigman and T. P. Walker, Phys. Rev. D 64 (2001) 123506; S. H. Hansen, G. Mangano, A. Melchiorri, G. Miele and O. Pisanti, Phys. Rev. D 65 (2002) 023511; S. Chandrasekhar, Proc. Roy. Soc. Lond. A 349 (1976) 571.

[2]D. Choudhary, N. D. Hari Dass, and M. V. N. Murthy, Class. Quantum. Grav. 6 (1989) L167.

[3]B. Mukhopadhyay, Class. Quantum Grav. 17 (2000) 2017; P. Singh and B. Mukhopadhyay, Mod. Phys. Lett. A 18 (2003) 779.

[4] H.Aissaoui, N.Mebarki and H.Bouhalouf , FRW Like Cosmological Model and Accelerated Expansion of the Universe from Non Commutative Seiberg-Witten Geometry, in proceedings of Third Algerian Workshop On Astronomy and Astrophysics, 12-13 June 2010, Constantine, Algeria, AIP Conf.Proc. 1295 (2010) 164.

[5] N. Mebarki, S. Zaim, L. Khodja and H. Aissaoui, Phys. Scripta 78 (2008) 045101.

[6]F. Khelili, J. Mimouni and N. Mebarki, J. Math. Phys. 42 (2007) 3615.

[7]N. Mebarki, F. Khelili, S.Kalli and M.Haouchine Chin. J. Phys. 44 (2008) 180.

[8]U. Debnath, B. Mukhopadhyay and N. Dadhich, Mod. Phys. Lett. A 21 (2006) 399. 